\title{
An Improvement in Power Management in green Computing using Neural Networks
}

\author{
Saket Bhushan ${ }^{1}$, Manoj Chaudhary ${ }^{2}$ \\ Student, Computer Science YCoE, Punjabi University, Punjab, India ${ }^{l}$ \\ Assistant Professor, Computer Science, YCoE, Punjabi University, Punjab, India ${ }^{2}$
}

\begin{abstract}
The green computing is the technology which is based on the environmental use of computer related resources. The computer related resources includes processing units, storage units etc. In such type of technology the energy consumption is the main concern. Green computing wills leads to reduce in resource consumption and electronic waste. The new technology of green computing will also include cloud computing services, grid computing services. In the past decades, many techniques have been proposed for the energy conservation in green computing. In this paper, we are proposing new technique for energy conservation in green computing. This novel technique is based on neural networks. The neural network is having capability of learning from the past experiences. The dynamic clustering approach is used with the neural networks for the energy conservation. The proposed technique is implemented in NS2 and simulation results are shown in the graphical form.
\end{abstract}

Keywords: Green computing, neural networks, dynamic clustering, learning

\section{Introduction}

The green computing is basically a technology which is based on the environmental responsible way to reduce power and environmental waste. In the present time, we are making efforts to make everything greener. This technology is related to reduce or managing impact of IT systems which basically includes materials and resources required for equipment, energy and also materials used in OS, potential health effects on humans from using equipment, and responsibility for the waste products that are created from IT systems. The green technology mainly focuses on super computers and cluster system which harm the green environment badly. The green computing is similar to green chemistry. In the approach of green computing we mainly focus on to design such systems which are having minimum effect to environment. The products supports reduce the use of hazardous materials, maximum energy efficient during the products lifetime and promote the recyclability of defunct products and factory waste. Artificial neural network is composed of interconnecting of artificial neurons. Artificial neural networks may either be used to gain understanding of biological neuron or to solve artificially intelligence problems without creating any model [8]. Biological neural networks are made up of real biological neurons that are connected or functionally related in nervous system [wiki].neural network has been motivated from human brain. The brain is highly complex, parallel computer and nonlinear. It has capability to organize its constituent's structure known as neurons to perform complex computations. It is faster than digital computer exit in today's world [8]. It is an adaptive in nature that changes its structure based on internal and external information that flows through the networks. Dynamic clustering is an energy efficient algorithm. Energy dissipation of the network can be reduced by using clustering algorithms. The energy consumption of nodes is depends upon the transmission distance, optimal routing protocols and amount of data to be transmitted. In cluster based networks, cluster heads $(\mathrm{CH})$ meets these requirements 1) same adjacent sensors are grouped into a cluster. 2) High energy resources 3) Network should be distributed.

\section{Litrature Review}

In the year 2012 R.Yamini "Power Management in Cloud Computing Using Green Algorithm" The greatest environmental challenge today is global warming, which is caused by carbon emissions. Energy crisis brings green computing, and green computing needs algorithms and mechanisms to be redesigned for energy efficiency. Green IT refers to the study and practice of using computing resources in an efficient, effective and economic way. The various approaches of the green IT are Virtualization, Power Management, Material Recycling and Telecommuting. The basic principles of cloud computing is to make the computing be assigned in a great number of distributed computers rather than local computer or remote server. In fact, cloud computing is an extend of Grid Computing, Distributed Computing and Parallel Computing. Thus, it is necessary to significantly reduce pollution and substantially lower energy usage. The analysis of energy consumption in cloud computing consider both public and private clouds. Cloud computing with green algorithm can enable more energy-efficient use of computing power[1]. 
In year 2010 Priya Rana "GREEN COMPUTING SAVES GREEN" Overview of the concept of Green Computing and how we can adopt it in our daily life to improve the deteriorating environmental conditions and how to adapt to sustainable. The understated data gives a basic computing. Green computing or green IT, refers to environmentally sustainable computing or IT whose goals are to reduce the use of hazardous materials, maximize energy efficiency during the product's lifetime, and promote the recyclability or biodegradability of defunct products and factory waste. Green Computing concentrates on energy efficiency, reducing resource consumption and disposing of electronic waste in a responsible manner. Green computing is the environmentally responsible use of computers and related resources. Such practices include the implementation of energy-efficient central processing units (CPUs), servers and peripherals as well as reduced resource consumption and proper disposal of electronic waste (e-waste). Computers today have become a necessity not only in offices but also at homes. Superficially, this can be called as Green Computing. In a deeper sense, Green Computing is the study which lays stress on the operation of computers and related peripherals in order to minimize the carbon footprint. Green computing strategies can help us to build a safe place for us to live in .If each one of us becomes successful in constructing a healthy environmental balcony for ourselves then trust us we can together make our mother earth a healthy and a happy place for us to survive here[2].

In year 2012 S.V.S.S. Lakshmi " A Study On Green Computing: The Future Computing And EcoFriendly Technology" Green computing, the study of efficient and eco-friendly computing resources, is under the attention of environmental organizations, and businesses from other industries. In recent years, companies in the computer industry have come to realize that going green is in their best interest, both in terms of public relations and reduced costs. This paper presents at several green initiatives currently under way in the computer industry, as well as issues that have been raised regarding these initiatives and presents a study with an example to learn more about the future of green computing[3].

Green computing or green IT, basically concerns to environmentally sustainable computing or IT. The field of green computing is defined as "the knowledge and practice of designing, manufacturing, using, and disposing of computers, servers, and associated subsystems - which include printers, monitors, and networking, storage devices and communications system efficiently and effectively with minimal or no impact on the environment. This computing is similar to green chemistry that is minimum utilization of hazardous materials and, maximizing energy efficiency during the product's lifetime, and also promote the recyclability or biodegradability of defunct products and factory waste [4].Green computing is raising energy costs and potential savings. Energy to manufacture, store, operate, and cool computing systems has grown significantly in the recent years, primarily due to the volume of systems and computing that companies now heavily rely upon. This paper describes about history of green computing, need of the green computing and future of the green computing. The primary objective of such a program is to account for the triple efficiently bottom line [5]. In today's eras the most every person is using computer. Every office is computerized and every one use the computer for their own purpose. But no one is aware but the harmful effects of the computer. The computes emit carbon dioxide harmful gases. The energy consumption by various computing devices is also plays a main role towards our harmful environment. This research paper analyzes the factors related to awareness of green computing among common man. It also includes a survey questionnaire which takes all the factors into account and before starting actual survey a pilot study for this questionnaire was done which confirm its acceptance [6].

A Dynamic Clustering-Based Algorithm for Wireless Sensor Networks, have discussed about energy aware Qos protocol for routing which are also efficient for best effort traffic . In this paper they explained how to improve first order energy efficiency by using dynamic clustering and to support Qos build multi-objective programming models [7].

\section{Problem Formulation}

Till yet researcher's research on various grids rebuilding algorithms which shows by it enhances network lifetime of an wireless sensors networks and even very much helpful in increasing the rate of dead sensors and avoiding the probability of pits, which results in increase of packet delivery time and decrease in packet loss rate. In a network there are number of nodes present in it. These nodes are then combined to form groups. These groups are known as clusters. Each cluster has only one controller in it. The communication with each node is controlled by cluster head and it also updates the nodes. This controller is known as cluster head. In an individual cluster only one cluster head is present. To communicate to one cluster to another cluster head is used. The nodes which are choose cluster head according to the election algorithm. It means the powerful node become cluster head. Cluster head is a winning node .The entire communication takes place with the help of these clusters only. There are number of clusters present in a network but each cluster has only one cluster head. The source is present which send data to destination through any route. Here destination means sink or base station. First of all the source send data to one of the cluster head of cluster. Then this cluster send data to another cluster and communication takes place source and sink. The shortest path should be choosing to send data from source to sink. We can say that the path which 
has minimum hop count that path is a shortest path to send data from source to sink. This is a dynamic process.

The cluster node if fails then another node can be chosen as a cluster head. By changing cluster head again and again may degrade the performance of the system. It means all the network change so it is not stable in nature. As the change of cluster heads topology also changes. Here in this work the main problem is energy consumption and the network lifetime. There are many methods to overcome this problem. Several energy conserving mechanisms have been proposed to extend the network lifetime of network and to improve the energy consumption problem. Nodes are not stable in it. To reduce energy consumption re- clustering in grids is required. Here our concern is to re-clustering the grids using dynamic clustering techniques. The clustering is used to minimize the energy consumption.

\section{Research Mythology}

There are number of nodes present in a network. These nodes participate to communicate with one another nodes. When all the nodes are combined then cluster are formed. So clustering is a combination of nodes. There is a controller of these nodes is also present. This controller is known as cluster head. In each cluster one cluster head is present. All the nodes in a cluster communicate with their cluster head. Then cluster head of a cluster further communicate with other cluster head to send data packets from source to destination.

Cluster head control all the activities done by the nodes in a cluster. A source sends information that is called source point and wants to send data to the sink which is base station or destination. The communication between sink to source is possible with the help of cluster head only.To choose the cluster head between numbers of nodes in a cluster is also an important task. The node in a cluster which consumes lowest battery power as compare to other nodes is select as cluster head. The cluster head is select according to the AODV algorithm. All the cluster head which participate in a selection of final path of route consume minimum battery power means have minimum energy consumption. If we select the cluster head which consume maximum battery then that cluster head will die very soon so all the system is effected with it and will dead soon. So the selection of cluster head is very important. The cluster head which will dead soon degrades the performance of the system.

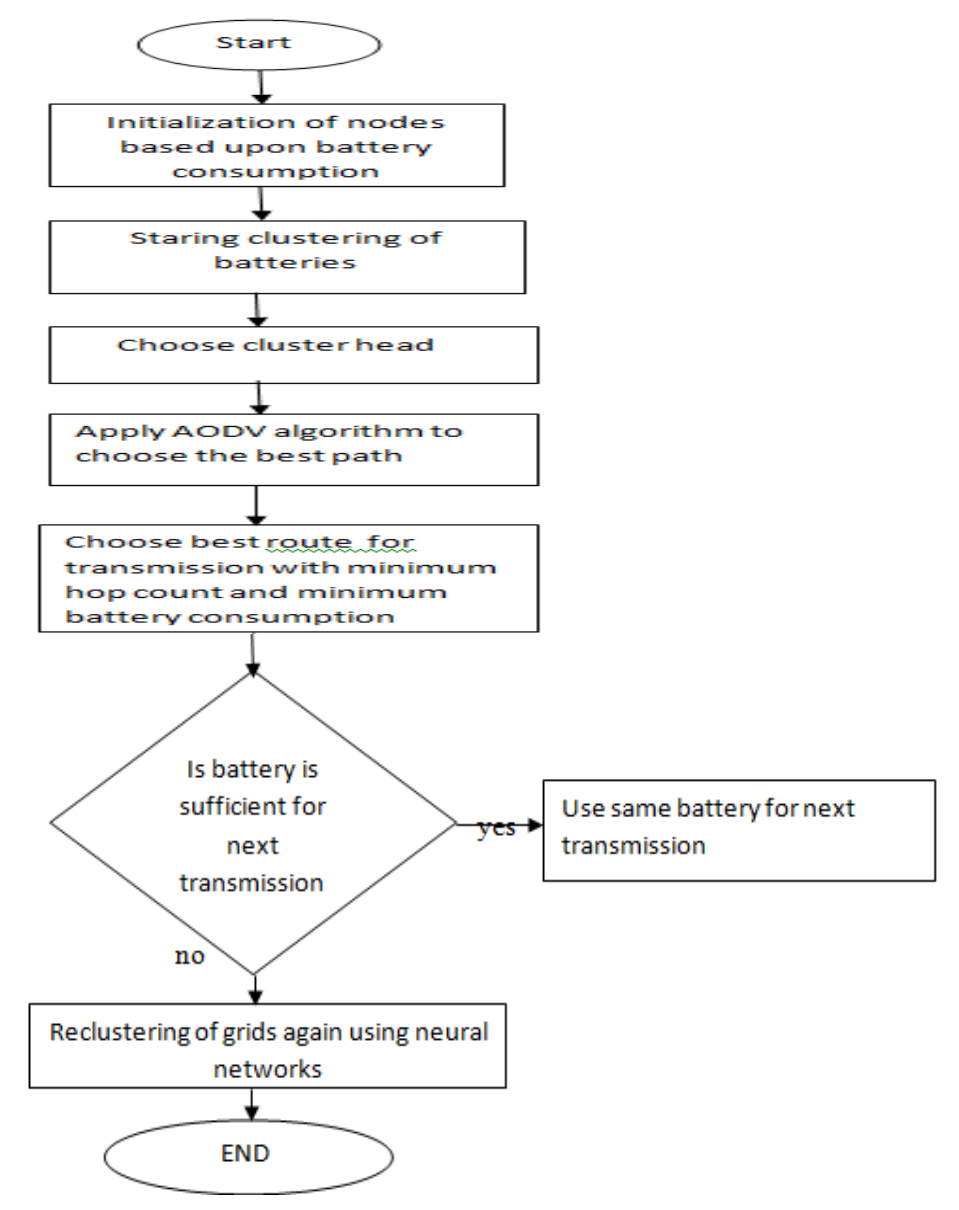

Figure 1 Flow Chart for the entire process 
Fully Network Deployment

\section{Results and Discussion}

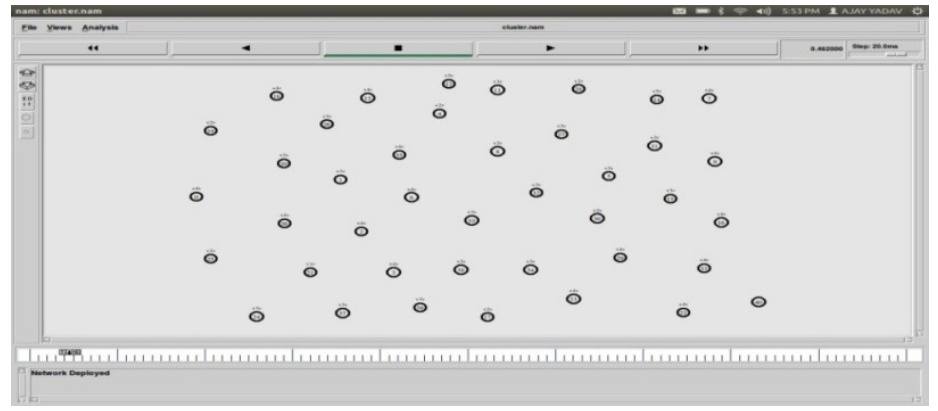

Figure 2 Snapshots of Implementation

All the nodes start communicate with their neighbor nodes and represent their battery resources

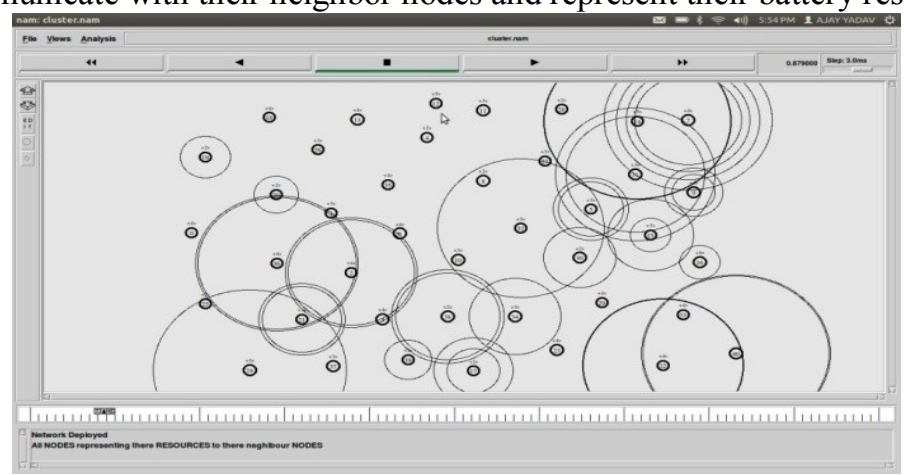

Figure 3 Snapshots of Implementation

Network rearrange and communication with neighbour node continuous

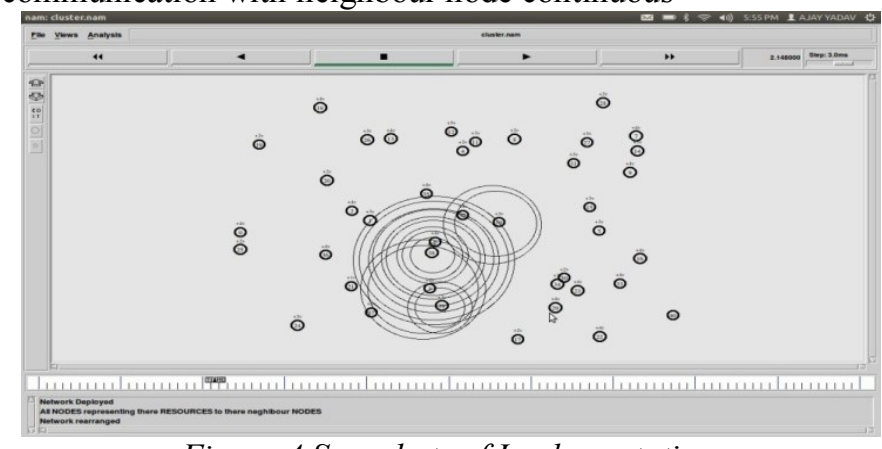

Figure 4 Snapshots of Implementation

Cluster formation start during the communication with neighbor node according to the battery capacity

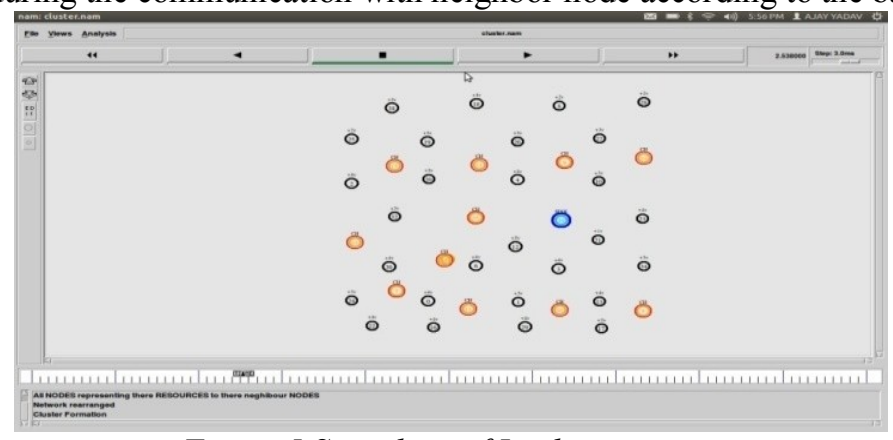

Figure 5 Snapshots of Implementation

Selection of cluster head of that node randomly after the rearrange of the network 


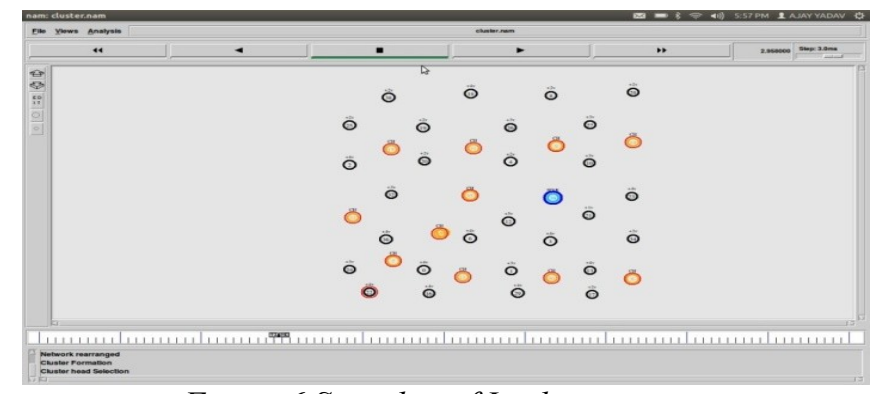

Figure 6 Snapshot of Implementation

Transmission starts after the cluster head selection

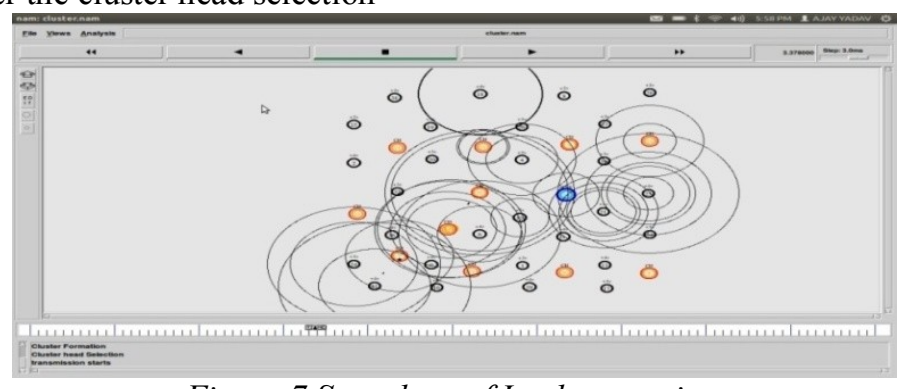

Figure 7 Snapshots of Implementation

At one cluster head battery low so link failure occure no further transmission possible packet loss

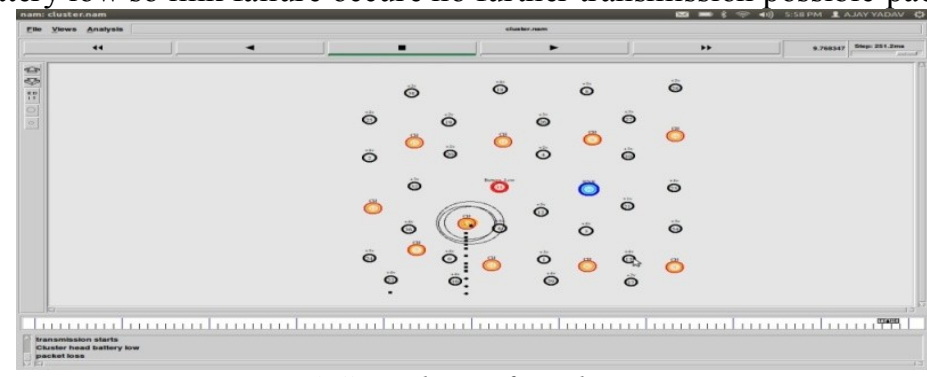

Figure 8 Snapshots of Implementation

Transmission starts between the cluster head

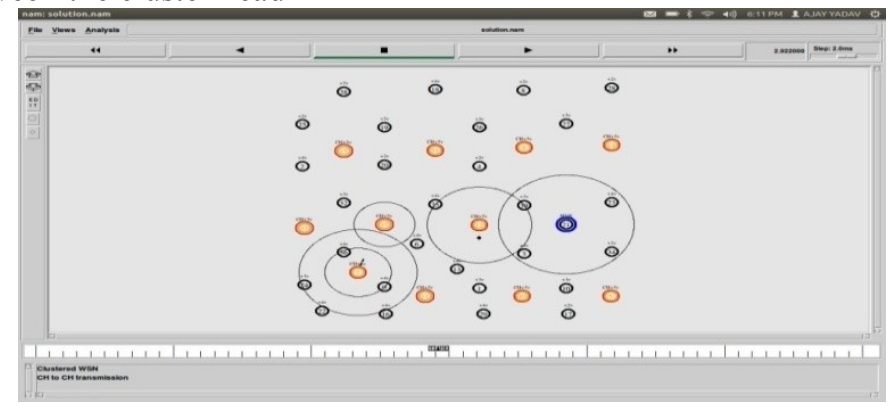

Figure 9 Snapshot of Implementation

Cluster head to cluster head transmission starts and path is select if battery Low then cluster and path both changed

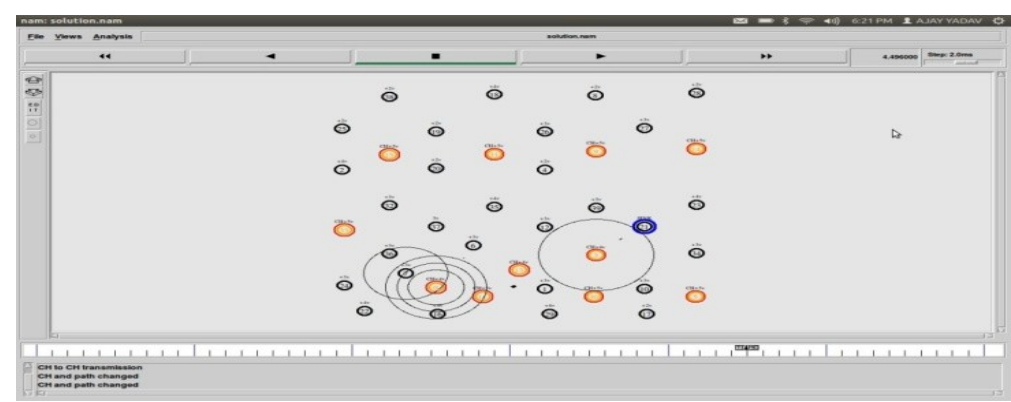

Figure 10 Snapshot of Implementation 


\subsection{Graphs}

\section{Solution has less delay as compared to the old method}

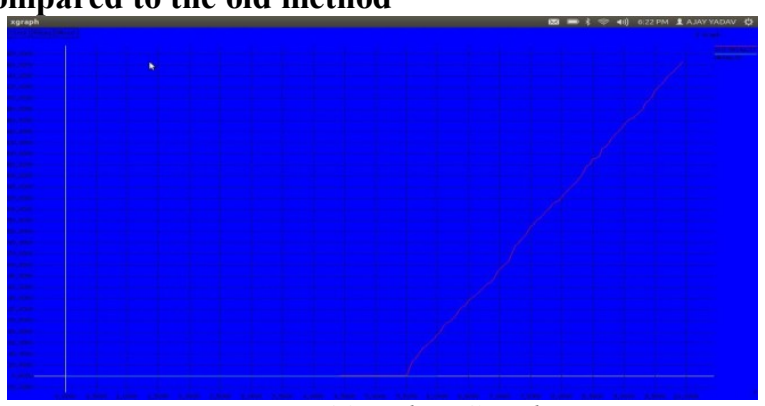

Figure 11 Delay Graph

Novel has less delay as compared to the old method. In this graph as the number of packets is increasing delay remains constant as compared with old delay.

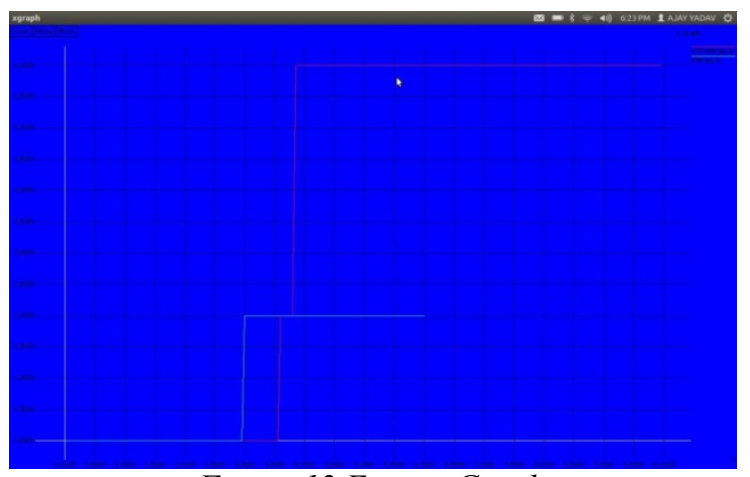

Figure 12 Energy Graph

The energy graph shows that with the implementation of novel approach, energy consumption is less as compared to the other existing approaches. The energy will be consumed less due to the dynamic clustering.

\section{Conclusion}

In this paper, we conclude that green computing is the emerging technology which will reduce IT waste. The main objective of this technology is to reduce the energy consumption of computer related products. In this we proposed new technique which is based on neural networks. The simulation results shows that it reduce battery consumption of computer devices.

\section{References}

[1] R.Yamini "Power Management in Cloud Computing Using Green Algorithm" IEEE-International Conference On Advances In Engineering, Science And Management (ICAESM -2012) March 30, 31, 2012.

[2] Rana "GREEN COMPUTING SAVES GREEN" International Journal of Advanced Computer and Mathematical Sciences. Vol 1, Issue 1, Dec, 2010, pp 45-51.

[3] S.V.S.S. Lakshmi “ A Study On Green Computing: The Future Computing And Eco-Friendly Technology” International Journal of Engineering Research and Applications (IJERA) ISSN: 2248-9622 Vol. 2, Issue4, July-August 2012, pp.1282-1285.

[4] Mujtaba Talebi and Thomas Way, "Methods, Metrics and Motivation for a Green Computer Science Program" SIGCSE'09, March 3-7, 2009, Chattanooga, Tennessee, USA" Copyright 2009 CM 978-1-60558-183-5/09/03...\$5.00

[5] Dr. Pardeep Mittal el al," Study and Analyze the Factors Related to Green Computing", International Journal of Advanced Research in Computer Science and Software Engineering 3(4),April - 2013, pp. 366-369

[6] Karsen Schmid," Green Compting A case for data caching and Flash Disks", University of Kaiserslautern, Gottlieb-Daimler-Str., 67663 Kaiserslautern, Germany

[7] Limin Meng, Kai Zhou, Jingyu Hua, Zhijiang Xu, "A Dynamic Clustering-Based Algorithm for Wireless Sensor Networks", International Symposium on Computer Science and Computational Technology,2008

[8] Eric Lee Eekhoff, "Wireless sensor networks and personal area networks for data integration in a virtual reality environment" 